\title{
EQUICONTINUITY OF MAPPINGS QUASICONFORMAL IN THE MEAN
}

\section{Vladimir Ryazanov and Evgeniı̌ Sevost'yanov}

National Academy of Sciences of Ukraine, Institute of Applied Mathematics and Mechanics 74 Roza Luksemburg Str., 83 114, Donetsk, Ukraine; vlryazanov1@rambler.ru

National Academy of Sciences of Ukraine, Institute of Applied Mathematics and Mechanics 74 Roza Luksemburg Str., 83 114, Donetsk, Ukraine; brusin2006@rambler.ru

\begin{abstract}
It is stated equicontinuity and normality of families $\mathfrak{R}^{\Phi}$ of the so-called $\operatorname{ring} Q(x)$ homeomorphisms with integral constraints of the type $\int \Phi(Q(x)) d m(x)<\infty$ in a domain $D \subset \mathbf{R}^{n}$, $n \geq 2$. It is shown that the found conditions on the function $\Phi$ are not only sufficient but also necessary for equicontinuity and normality of such families of mappings. It is also given applications of these results to families of mappings in the Sobolev class $W_{\text {loc }}^{1, n}$.
\end{abstract}

\section{Introduction}

Here $d m(x)$ corresponds to the Lebesgue measure in a domain $D$ in $\mathbf{R}^{n}, n \geq 2$. In the theory of mappings called quasiconformal in the mean, conditions of the type

$$
\int_{D} \Phi(Q(x)) d m(x)<\infty
$$

are standard for various characteristics $Q$ of these mappings, see e.g. [1], [3], [7], [8], [11]-[15], [18], [19], [21] and [25]. The study of classes with the integral conditions (1.1) is also actual in the connection with the recent development of the theory of degenerate Beltrami equations and the so-called mappings with finite distortion, see e.g. related references in the monographs [9] and [17].

In the present paper we study the problems of equicontinuity and normality for wide classes of the so-called ring $Q(x)$-homeomorphisms with the condition (1.1) and give the corresponding applications to Sobolev's classes.

Recall that the (conformal) modulus of a family $\Gamma$ of curves $\gamma$ in $\mathbf{R}^{n}, n \geq 2$, is the quantity

$$
M(\Gamma)=\inf _{\rho \in \operatorname{adm} \Gamma} \int_{\mathbf{R}^{n}} \rho^{n}(x) d m(x)
$$

where a Borel function $\rho: \mathbf{R}^{n} \rightarrow[0, \infty]$ is admissible for $\Gamma$, write $\rho \in \operatorname{adm} \Gamma$, if

$$
\int_{\gamma} \rho(x)|d x| \geq 1 \quad \forall \gamma \in \Gamma .
$$

doi:10.5186/aasfm.2011.3614

2010 Mathematics Subject Classification: Primary 30C65, 30C75.

Key words: Equicontinuity, normality, quasiconformal, integral constraints, Sobolev classes. 
One of the equivalent geometric definitions of $K$-quasiconformal mappings $f$ with $K \in[1, \infty)$ given in a domain $D$ in $\mathbf{R}^{n}, n \geq 2$, is reduced to the inequality

$$
M(f \Gamma) \leq K M(\Gamma)
$$

that holds for an arbitrary family $\Gamma$ of curves $\gamma$ in the domain $D$. Similarly, given a domain $D$ in $\mathbf{R}^{n}, n \geq 2$, and a (Lebesgue) measurable function $Q: D \rightarrow[1, \infty]$, a homeomorphism $f: D \rightarrow \overline{\mathbf{R}^{n}}, \overline{\mathbf{R}^{n}}=\mathbf{R}^{n} \cup\{\infty\}$, is called $Q(x)$-homeomorphism if

$$
M(f \Gamma) \leq \int_{D} Q(x) \cdot \rho^{n}(x) d m(x)
$$

for every family $\Gamma$ of curves $\gamma$ in $D$ and every $\rho \in \operatorname{adm} \Gamma$, see e.g. [17].

In the case $Q(x) \leq K$ a.e., we again come to the inequality (1.2). In the general case, the latter inequality means that the conformal modulus of the family $f \Gamma$ is estimated by the modulus $M_{Q}$ of $\Gamma$ with the weight $Q, M(f \Gamma) \leq M_{Q}(\Gamma)$, see e.g. [2]. The inequality of the type (1.3) was first stated by Lehto and Virtanen for quasiconformal mappings in the plane, see Section V.6.3 in [16]. The relation of the type (1.3) was also stated by Bishop, Gutlyanskii, Martio and Vuorinen in [4] for quasiconformal mappings in space where $Q(x)$ is equal to $K_{I}(x, f)$.

Recall that the inner dilatation of a mapping $f: D \rightarrow \mathbf{R}^{n}, n \geq 2$, at a point $x \in D$ of differentiability for $f$ is

$$
K_{I}(x, f)=\frac{|J(x, f)|}{l\left(f^{\prime}(x)\right)^{n}}
$$

if $J(x, f) \neq 0, K_{I}(x, f)=1$ if $f^{\prime}(x)=0$, and $K_{I}(x, f)=\infty$ at the rest points, where $J(x, f)$ is the Jacobian of $f$ at $x$ and

$$
l\left(f^{\prime}(x)\right)=\inf _{h \in \mathbf{R}^{n} \backslash\{0\}} \frac{\left|f^{\prime}(x) h\right|}{|h|} .
$$

The following notion generalizes and localizes the above notion of a $Q$-homeomorphism. It is motivated by the ring definition of Gehring for quasiconformal mappings, see e.g. [6], introduced first in the plane, see [23], and extended later on to the space case in [22], see also Chapters 7 and 11 in [17]. Let $E, F \subset \overline{\mathbf{R}^{n}}$ be arbitrary sets. Denote by $\Gamma(E, F, D)$ a family of all curves $\gamma:[a, b] \rightarrow \overline{\mathbf{R}^{n}}$ joining $E$ and $F$ in $D$, i.e. $\gamma(a) \in E, \gamma(b) \in F$ and $\gamma(t) \in D$ as $t \in(a, b)$.

Given a domain $D$ in $\mathbf{R}^{n}, n \geq 2$, a (Lebesgue) measurable function $Q: D \rightarrow$ $[0, \infty], x_{0} \in D$, a homeomorphism $f: D \rightarrow \overline{\mathbf{R}^{n}}$ is said to be a ring $Q$-homeomorphism at the point $x_{0}$ if

$$
M\left(f\left(\Gamma\left(S_{1}, S_{2}, R\right)\right)\right) \leq \int_{R} Q(x) \cdot \eta^{n}\left(\left|x-x_{0}\right|\right) d m(x)
$$

for every ring $R=R\left(r_{1}, r_{2}, x_{0}\right)=\left\{x \in \mathbf{R}^{n}: r_{1}<\left|x-x_{0}\right|<r_{2}\right\}$ and the spheres $S_{i}=S\left(x_{0}, r_{i}\right)=\left\{x \in \mathbf{R}^{n}:\left|x-x_{0}\right|=r_{i}\right\}$, where $0<r_{1}<r_{2}<r_{0}:=\operatorname{dist}\left(x_{0}, \partial D\right)$, and every measurable function $\eta:\left(r_{1}, r_{2}\right) \rightarrow[0, \infty]$ such that

$$
\int_{r_{1}}^{r_{2}} \eta(r) d r \geq 1
$$


Moreover, $f$ is called a ring $Q$-homeomorphism in the domain $D$ if $f$ is a $\operatorname{ring} Q$ homeomorphism at every point $x_{0} \in D$. Note that, in particular, homeomorphisms $f: D \rightarrow \mathbf{R}^{n}$ in the class $W_{\text {loc }}^{1, n}$ with $K_{I}(x, f) \in L_{\text {loc }}^{1}$ are ring $Q$-homeomorphisms with $Q(x)=K_{I}(x, f)$, see e.g. Theorem 4.1 in [17].

The notion of ring $Q$-homeomorphism can be extended in the natural way to $\infty$. More precisely, under $\infty \in D \subseteq \overline{\mathbf{R}^{n}}$ a homeomorphism $f: D \rightarrow \overline{\mathbf{R}^{n}}$ is called a ring $Q$-homeomorphism at $\infty$ if the mapping $\widetilde{f}=f\left(\frac{x}{|x|^{2}}\right)$ is a ring $Q^{\prime}$-homeomorphism at the origin with $Q^{\prime}(x)=Q\left(\frac{x}{|x|^{2}}\right)$. In other words, a mapping $f: D \rightarrow \overline{\mathbf{R}^{n}}$ is a ring $Q$-homeomorphism at $\infty$ iff

$$
M\left(f\left(\Gamma\left(S\left(R_{1}\right), S\left(R_{2}\right), R\right)\right)\right) \leq \int_{R} Q(y) \cdot \eta^{n}(|y|) d m(y)
$$

holds for every ring $R=R\left(R_{1}, R_{2}, 0\right)=\left\{y \in \mathbf{R}^{n}: R_{1}<|y|<R_{2}\right\}$ in $D$ with $0<R_{1}<R_{2}<\infty, S\left(R_{i}\right)=\left\{x \in \mathbf{R}^{n}:|x|=R_{i}\right\}$ and for every measurable function $\eta:\left(R_{1}, R_{2}\right) \rightarrow[0, \infty]$ with $\int_{R_{1}}^{R_{2}} \eta(r) d r \geq 1$.

\section{Preliminaries}

Let $(X, d)$ and $\left(X^{\prime}, d^{\prime}\right)$ be metric spaces with distances $d$ and $d^{\prime}$, respectively. A family $\mathfrak{F}$ of continuous mappings from $X$ into $X^{\prime}$ is said to be a normal if every sequence of mappings $f_{m}$ in $\mathfrak{F}$ has a subsequence $f_{m_{k}}$ converging to a continuous mapping $f: X \rightarrow X^{\prime}$ uniformly on each compact set $C \subset X$. Normality is closely related to the following notion. A family $\mathfrak{F}$ of mappings $f: X \rightarrow X^{\prime}$ is said to be equicontinuous at a point $x_{0} \in X$ if for every $\varepsilon>0$ there is $\delta>0$ such that $d^{\prime}\left(f(x), f\left(x_{0}\right)\right)<\varepsilon$ for all $f \in \mathfrak{F}$ and $x \in X$ with $d\left(x, x_{0}\right)<\delta$. The family $\mathfrak{F}$ is called equicontinuous if $\mathfrak{F}$ is equicontinuous at every point $x_{0} \in X$. The following version of the Arzela-Ascoli theorem will be useful later on, see e.g. Section 20.4 in [26].

Proposition 2.1. Let $(X, d)$ be a separable metric space and let $\left(X^{\prime}, d^{\prime}\right)$ be a compact metric space. Then a family $\mathfrak{F}$ of mappings $f: X \rightarrow X^{\prime}$ is normal if and only if $\mathfrak{F}$ is equicontinuous.

In particular, Proposition 2.1 holds in the case when $X=\mathbf{R}^{n}$ with the usual distance and $X^{\prime}$ is the extended space $\overline{\mathbf{R}^{n}}=\mathbf{R}^{n} \cup\{\infty\}$ (compact) with the spherical metric. Recall that the spherical (chordal) metric $h(x, y)$ in $\overline{\mathbf{R}^{n}}$ is equal to $\mid \pi(x)-$ $\pi(y) \mid$ where $\pi$ is the stereographic projection of $\overline{\mathbf{R}^{n}}$ on the sphere $S^{n}\left(\frac{1}{2} e_{n+1}, \frac{1}{2}\right)$ in $\mathbf{R}^{n+1}$, i.e., in the explicit form,

$$
h(x, \infty)=\frac{1}{\sqrt{1+|x|^{2}}}, \quad h(x, y)=\frac{|x-y|}{\sqrt{1+|x|^{2}} \sqrt{1+|y|^{2}}}, \quad x \neq \infty \neq y .
$$

The spherical diameter of a set $E$ in $\overline{\mathbf{R}^{n}}$ is the quantity $h(E)=\sup _{x_{1}, x_{2} \in E} h\left(x_{1}, x_{2}\right)$.

Let $\mathfrak{R}_{Q, \Delta}(D)$ be the class of all ring $Q$-homeomorphisms $f$ in a domain $D \subseteq \mathbf{R}^{n}$, $n \geq 2$, such that $h\left(\overline{\mathbf{R}^{n}} \backslash f(D)\right) \geq \Delta>0$. The following distortion estimate under $Q$-homeomorphisms can be found in [22], see also Theorem 7.3 in [17]. 
Proposition 2.2. Let $\Delta>0, Q: D \rightarrow[0, \infty]$ be a measurable function. Then

$$
h\left(f(x), f\left(x_{0}\right)\right) \leq \frac{\alpha_{n}}{\Delta} \exp \left\{-\int_{\left|x-x_{0}\right|}^{\varepsilon\left(x_{0}\right)} \frac{d r}{r q_{x_{0}}^{\frac{1}{n-1}}(r)}\right\}
$$

for every $f \in \mathfrak{R}_{Q, \Delta}(D)$ and $x \in B\left(x_{0}, \varepsilon\left(x_{0}\right)\right), \varepsilon\left(x_{0}\right)<\operatorname{dist}\left(x_{0}, \partial D\right)$, where $\alpha_{n}>0$ depends only on $n$ and $q_{x_{0}}(r)$ is the mean value of the function $Q$ over the sphere $\left|z-x_{0}\right|=r$.

For every non-decreasing function $\Phi:[0, \infty] \rightarrow[0, \infty]$, the inverse function $\Phi^{-1}$ : $[0, \infty] \rightarrow[0, \infty]$ can be well defined by setting

$$
\Phi^{-1}(\tau)=\inf _{\Phi(t) \geq \tau} t
$$

As usual, here inf is equal to $\infty$ if the set of $t \in[0, \infty]$ such that $\Phi(t) \geq \tau$ is empty. Note that the function $\Phi^{-1}$ is non-decreasing, too.

Remark 2.1. Immediately by the definition it is evident that

$$
\Phi^{-1}(\Phi(t)) \leq t \quad \forall t \in[0, \infty]
$$

with the equality in (2.3) except intervals of constancy of the function $\Phi(t)$.

Since the mapping $t \mapsto t^{p}$ for every positive $p$ is a sense-preserving homeomorphism $[0, \infty]$ onto $[0, \infty]$, we may rewrite Theorem 2.1 from [24] in the following form which is more convenient for further applications. Here, in (2.5) and (2.6), we complete the definition of integrals by $\infty$ if $\Phi_{p}(t)=\infty$, correspondingly, $H_{p}(t)=\infty$ for all $t \geq T \in[0, \infty)$. The integral in (2.6) is understood as the Lebesgue-Stieltjes integral and the integrals in (2.5) and (2.7)-(2.10) as the ordinary Lebesgue integrals.

Proposition 2.3. Let $\Phi:[0, \infty] \rightarrow[0, \infty]$ be a non-decreasing function. Set

$$
H_{p}(t)=\log \Phi_{p}(t), \quad \Phi_{p}(t)=\Phi\left(t^{p}\right), \quad p \in(0, \infty) .
$$

Then the equality

$$
\int_{\delta}^{\infty} H_{p}^{\prime}(t) \frac{d t}{t}=\infty
$$

implies the equality

$$
\int_{\delta}^{\infty} \frac{d H_{p}(t)}{t}=\infty
$$

and (2.6) is equivalent to

$$
\int_{\delta}^{\infty} H_{p}(t) \frac{d t}{t^{2}}=\infty
$$

for some $\delta>0$, and (2.7) is equivalent to every of the equalities:

$$
\int_{0}^{\Delta} H_{p}\left(\frac{1}{t}\right) d t=\infty
$$


for some $\Delta>0$,

$$
\int_{\delta_{*}}^{\infty} \frac{d \eta}{H_{p}^{-1}(\eta)}=\infty
$$

for some $\delta_{*}>H(+0)$,

$$
\int_{\delta_{*}}^{\infty} \frac{d \tau}{\tau \Phi_{p}^{-1}(\tau)}=\infty
$$

for some $\delta_{*}>\Phi(+0)$.

Moreover, (2.5) is equivalent to (2.6) and hence (2.5)-(2.10) are equivalent each to other if $\Phi$ is in addition absolutely continuous. In particular, all the conditions (2.5)-(2.10) are equivalent if $\Phi$ is convex and non-decreasing.

It is easy to see that conditions $(2.5)-(2.10)$ become weaker as $p$ increases, see e.g. (2.7). It is necessary to give one more explanation. From the right hand sides in the conditions $(2.5)-(2.10)$ we have in mind $+\infty$. If $\Phi_{p}(t)=0$ for $t \in\left[0, t_{*}\right]$, then $H_{p}(t)=-\infty$ for $t \in\left[0, t_{*}\right]$ and we complete the definition $H_{p}^{\prime}(t)=0$ for $t \in\left[0, t_{*}\right]$. Note, the conditions (2.6) and (2.7) exclude that $t_{*}$ belongs to the interval of integrability because in the contrary case the left hand sides in (2.6) and (2.7) are either equal to $-\infty$ or indeterminate. Hence we may assume in (2.5)-(2.8) that $\delta>t_{0}$, correspondingly, $\Delta<1 / t_{0}$ where $t_{0}:=\sup _{\Phi_{p}(t)=0} t, t_{0}=0$ if $\Phi_{p}(0)>0$.

\section{The main lemma and its corollaries}

Recall that a function $\Phi:[0, \infty] \rightarrow[0, \infty]$ is called convex if

$$
\Phi\left(\lambda t_{1}+(1-\lambda) t_{2}\right) \leq \lambda \Phi\left(t_{1}\right)+(1-\lambda) \Phi\left(t_{2}\right)
$$

for all $t_{1}$ and $t_{2} \in[0, \infty]$ and $\lambda \in[0,1]$. In what follows, $\mathbf{R}^{n}(\varepsilon), \varepsilon \in(0,1)$, denotes the ring in the space $\mathbf{R}^{n}, n \geq 2$,

$$
\mathbf{R}^{n}(\varepsilon)=\left\{x \in \mathbf{R}^{n}: \varepsilon<|x|<1\right\} .
$$

The following statement is a generalization and strengthening of Lemma 3.1 from [24].

Lemma 3.1. Let $Q: \mathbf{B}^{n} \rightarrow[0, \infty]$ be a measurable function and let $\Phi:[0, \infty] \rightarrow$ $(0, \infty]$ be a non-decreasing convex function. Suppose that the mean value $M(\varepsilon)$ of the function $\Phi \circ Q$ over the ring $\mathbf{R}^{n}(\varepsilon), \varepsilon \in(0,1)$, is finite. Then

$$
\int_{\varepsilon}^{1} \frac{d r}{r q^{\frac{1}{p}}(r)} \geq \frac{1}{n} \int_{e M(\varepsilon)}^{\frac{M(\varepsilon)}{\varepsilon^{n}}} \frac{d \tau}{\tau\left[\Phi^{-1}(\tau)\right]^{\frac{1}{p}}} \quad \forall p \in(0, \infty)
$$

where $q(r)$ is the average of the function $Q(x)$ over the sphere $|x|=r$.

Remark 3.1. Note that (3.2) is equivalent for each $p \in(0, \infty)$ to the inequality

$$
\int_{\varepsilon}^{1} \frac{d r}{r q^{\frac{1}{p}}(r)} \geq \frac{1}{n} \int_{e M(\varepsilon)}^{\frac{M(\varepsilon)}{\varepsilon^{n}}} \frac{d \tau}{\tau \Phi_{p}^{-1}(\tau)}, \quad \Phi_{p}(t):=\Phi\left(t^{p}\right) .
$$


Note also that $M(\varepsilon)$ converges as $\varepsilon \rightarrow 0$ to the average of $\Phi \circ Q$ over the unit ball $\mathbf{B}^{n}$.

Proof. Denote $t_{*}=\sup _{\Phi_{p}(t)=\tau_{0}} t, \tau_{0}=\Phi(0)$. Setting $H_{p}(t)=\log \Phi_{p}(t)$, we see that $H_{p}^{-1}(\eta)=\Phi_{p}^{-1}\left(e^{\eta}\right), \Phi_{p}^{-1}(\tau)=H_{p}^{-1}(\log \tau)$. Thus, we obtain that

$$
q^{\frac{1}{p}}(r)=H_{p}^{-1}\left(\log \frac{h(r)}{r^{n}}\right)=H_{p}^{-1}\left(n \log \frac{1}{r}+\log h(r)\right) \quad \forall r \in R_{*}
$$

where $h(r):=r^{n} \Phi(q(r))=r^{n} \Phi_{p}\left(q^{\frac{1}{p}}(r)\right)$ and $R_{*}=\left\{r \in(\varepsilon, 1): q^{\frac{1}{p}}(r)>t_{*}\right\}$. Then also

$$
q^{\frac{1}{p}}\left(e^{-s}\right)=H_{p}^{-1}\left(n s+\log h\left(e^{-s}\right)\right) \quad \forall s \in S_{*}
$$

where $S_{*}=\left\{s \in\left(0, \log \frac{1}{\varepsilon}\right): q^{\frac{1}{p}}\left(e^{-s}\right)>t_{*}\right\}$.

Now, by the Jensen inequality and convexity of $\Phi$ we have that

$$
\begin{aligned}
\int_{0}^{\log \frac{1}{\varepsilon}} h\left(e^{-s}\right) d s & =\int_{\varepsilon}^{1} h(r) \frac{d r}{r}=\int_{\varepsilon}^{1} \Phi(q(r)) r^{n-1} d r \leq \int_{\varepsilon}^{1}\left(\int_{S(r)} \Phi(Q(x)) d \mathscr{A}\right) r^{n-1} d r \\
& \leq \frac{\Omega_{n}}{\omega_{n-1}} \cdot M(\varepsilon)=\frac{1}{n} \cdot M(\varepsilon)
\end{aligned}
$$

where we use the mean value of the function $\Phi \circ Q$ over the sphere $S(r)=\{x \in$ $\left.\mathbf{R}^{n}:|x|=r\right\}$ with respect to the area measure. As usual, here $\Omega_{n}$ and $\omega_{n-1}$ is the volume of the unit ball and the area of the unit sphere in $\mathbf{R}^{n}$, correspondingly. Then arguing by contradiction it is easy to see that

$$
|T|=\int_{T} d s \leq \frac{1}{n}
$$

where $T=\left\{s \in\left(0, \log \frac{1}{\varepsilon}\right): h\left(e^{-s}\right)>M(\varepsilon)\right\}$. Next, let us show that

$$
q^{\frac{1}{p}}\left(e^{-s}\right) \leq H_{p}^{-1}(n s+\log M(\varepsilon)) \quad \forall s \in\left(0, \log \frac{1}{\varepsilon}\right) \backslash T_{*}
$$

where $T_{*}=T \cap S_{*}$. Note that $\left(0, \log \frac{1}{\varepsilon}\right) \backslash T_{*}=\left[\left(0, \log \frac{1}{\varepsilon}\right) \backslash S_{*}\right] \cup\left[\left(0, \log \frac{1}{\varepsilon}\right) \backslash T\right]=$ $\left[\left(0, \log \frac{1}{\varepsilon}\right) \backslash S_{*}\right] \cup\left[S_{*} \backslash T\right]$. The inequality (3.6) holds for $s \in S_{*} \backslash T$ by (3.4) because $H_{p}^{-1}$ is a non-decreasing function. Note also that $e^{n s} M(\varepsilon)>\Phi(0)=\tau_{0}$ for all $s \in(0, \log 1 / \varepsilon)$ and then $t_{*}<\Phi_{p}^{-1}\left(e^{n s} M(\varepsilon)\right)=H_{p}^{-1}(n s+\log M(\varepsilon))$ for all $s \in$ $(0, \log 1 / \varepsilon)$. Consequently, (3.6) holds for $s \in\left(0, \log \frac{1}{\varepsilon}\right) \backslash S_{*}$, too.

Since $H_{p}^{-1}$ is non-decreasing, we have by (3.5) and (3.6) that

$$
\begin{aligned}
\int_{\varepsilon}^{1} \frac{d r}{r q^{\frac{1}{p}}(r)} & =\int_{0}^{\log \frac{1}{\varepsilon}} \frac{d s}{q^{\frac{1}{p}}\left(e^{-s}\right)} \geq \int_{\left(0, \log \frac{1}{\varepsilon}\right) \backslash T_{*}} \frac{d s}{H_{p}^{-1}(n s+\Delta)} \geq \int_{\left|T_{*}\right|}^{\log \frac{1}{\varepsilon}} \frac{d s}{H_{p}^{-1}(n s+\Delta)} \\
& \geq \int_{\frac{1}{n}}^{\log \frac{1}{\varepsilon}} \frac{d s}{H_{p}^{-1}(n s+\Delta)}=\frac{1}{n} \int_{1+\Delta}^{n \log \frac{1}{\varepsilon}+\Delta} \frac{d \eta}{H_{p}^{-1}(\eta)}
\end{aligned}
$$


where $\Delta=\log M(\varepsilon)$. Note that $1+\Delta=\log e M(\varepsilon)$. Thus,

$$
\int_{\varepsilon}^{1} \frac{d r}{r q^{\frac{1}{p}}(r)} \geq \frac{1}{n} \int_{\log e M(\varepsilon)}^{\log \frac{M(\varepsilon)}{\varepsilon^{n}}} \frac{d \eta}{H_{p}^{-1}(\eta)}
$$

and, after the replacement $\eta=\log \tau$, we obtain (3.3) and hence (3.2).

Corollary 3.1. Let $\Phi:[0, \infty] \rightarrow(0, \infty]$ be a non-decreasing convex function, $Q: \mathbf{B}^{n} \rightarrow[0, \infty]$ a measurable function, $Q_{*}(x)=1$ if $Q(x)<1$, and $Q_{*}(x)=Q(x)$ if $Q(x) \geq 1$. Suppose that the mean $M_{*}(\varepsilon)$ of the function $\Phi \circ Q$ over the ring $\mathbf{R}^{n}(\varepsilon)$, $\varepsilon \in(0,1)$, is finite. Then

$$
\int_{\varepsilon}^{1} \frac{d r}{r q^{\frac{\lambda}{p}}(r)} \geq \frac{1}{n} \int_{e M_{*}(\varepsilon)}^{\frac{M_{*}(\varepsilon)}{\varepsilon^{n}}} \frac{d \tau}{\tau\left[\Phi^{-1}(\tau)\right]^{\frac{1}{p}}} \quad \forall \lambda \in(0,1), \quad p \in(0, \infty),
$$

where $q(r)$ is the average of the function $Q(x)$ over the sphere $|x|=r$.

Indeed, let $q_{*}(r)$ be the average of the function $Q_{*}(x)$ over the sphere $|x|=r$. Then $q(r) \leq q_{*}(r)$ and, moreover, $q_{*}(r) \geq 1$ for all $r \in(0,1)$. Thus, $q^{\frac{\lambda}{p}}(r) \leq q_{*}^{\frac{\lambda}{p}}(r) \leq$ $q_{*}^{\frac{1}{p}}(r)$ for all $\lambda \in(0,1)$, and hence by Lemma 3.1 applied to $Q_{*}(x)$ we obtain $(3.9)$.

Theorem 3.1. Let $Q: \mathbf{B}^{n} \rightarrow[0, \infty]$ be a measurable function such that

$$
\int_{\mathbf{B}^{n}} \Phi(Q(x)) d m(x)<\infty
$$

where $\Phi:[0, \infty] \rightarrow[0, \infty]$ is a non-decreasing convex function such that

$$
\int_{\delta_{0}}^{\infty} \frac{d \tau}{\tau\left[\Phi^{-1}(\tau)\right]^{\frac{1}{p}}}=\infty, \quad p \in(0, \infty)
$$

for some $\delta_{0}>\tau_{0}:=\Phi(0)$. Then

$$
\int_{0}^{1} \frac{d r}{r q^{\frac{1}{p}}(r)}=\infty
$$

where $q(r)$ is the average of the function $Q(x)$ over the sphere $|x|=r$. that

Remark 3.2. Since $\left[\Phi^{-1}(\tau)\right]^{\frac{1}{p}}=\Phi_{p}^{-1}(\tau)$ where $\Phi_{p}(t)=\Phi\left(t^{p}\right),(3.11)$ implies

$$
\int_{\delta}^{\infty} \frac{d \tau}{\tau \Phi_{p}^{-1}(\tau)}=\infty \quad \forall \delta \in[0, \infty)
$$

but (3.13) for some $\delta \in[0, \infty)$, generally speaking, does not imply (3.11). Indeed, for $\delta \in\left[0, \delta_{0}\right),(3.11)$ evidently implies $(3.13)$ and, for $\delta \in\left(\delta_{0}, \infty\right)$, we have that

$$
0 \leq \int_{\delta_{0}}^{\delta} \frac{d \tau}{\tau \Phi_{p}^{-1}(\tau)} \leq \frac{1}{\Phi_{p}^{-1}\left(\delta_{0}\right)} \log \frac{\delta}{\delta_{0}}<\infty,
$$


because $\Phi_{p}^{-1}$ is non-decreasing and $\Phi_{p}^{-1}\left(\delta_{0}\right)>0$. Moreover, by the definition of the inverse function $\Phi_{p}^{-1}(\tau) \equiv 0$ for all $\tau \in\left[0, \tau_{0}\right], \tau_{0}=\Phi_{p}(0)$, and hence (3.13) for $\delta \in\left[0, \tau_{0}\right)$, generally speaking, does not imply (3.11). If $\tau_{0}>0$, then

$$
\int_{\delta}^{\tau_{0}} \frac{d \tau}{\tau \Phi_{p}^{-1}(\tau)}=\infty \quad \forall \delta \in\left[0, \tau_{0}\right) .
$$

However, (3.15) gives no information on the function $Q(x)$ itself and, consequently, (3.13) for $\delta<\Phi(0)$ cannot imply (3.12) at all.

In view of (3.13), Theorem 3.1 follows immediately from Lemma 3.1.

Corollary 3.2. If $\Phi:[0, \infty] \rightarrow[0, \infty]$ is a non-decreasing convex function and $Q$ satisfies the condition (3.10), then each of the conditions (2.5)-(2.10) for $p \in(0, \infty)$ implies (3.12). Moreover, if in addition $\Phi(1)<\infty$ or $q(r) \geq 1$ on a subset of $(0,1)$ of a positive measure, then each of the conditions (2.5)-(2.10) for $p \in(0, \infty)$ implies

$$
\int_{0}^{1} \frac{d r}{r q^{\frac{\lambda}{p}}(r)}=\infty \quad \forall \lambda \in(0,1)
$$

and also

$$
\int_{0}^{1} \frac{d r}{r^{\alpha} q^{\frac{\beta}{p}}(r)}=\infty \quad \forall \alpha \geq 1, \beta \in(0, \alpha] .
$$

\section{Sufficient conditions for equicontinuity}

Let $D$ be a fixed domain in the extended space $\overline{\mathbf{R}^{n}}=\mathbf{R}^{n} \cup\{\infty\}, n \geq 2$. Given a function $\Phi:[0, \infty] \rightarrow[0, \infty], M>0, \Delta>0, \mathfrak{R}_{M, \Delta}^{\Phi}$ denotes the collection of all ring $Q(x)$-homeomorphisms in $D$ such that $h\left(\overline{\mathbf{R}^{n}} \backslash f(D)\right) \geq \Delta$ and

$$
\int_{D} \Phi(Q(x)) \frac{d m(x)}{\left(1+|x|^{2}\right)^{n}} \leq M .
$$

Theorem 4.1. Let $\Phi:[0, \infty] \rightarrow[0, \infty]$ be non-decreasing convex function. If

$$
\int_{\delta_{0}}^{\infty} \frac{d \tau}{\tau\left[\Phi^{-1}(\tau)\right]^{\frac{1}{n-1}}}=\infty
$$

for some $\delta_{0}>\tau_{0}:=\Phi(0)$, then the class $\mathfrak{R}_{M, \Delta}^{\Phi}$ is equicontinuous and, consequently, forms a normal family of mappings for every $M \in(0, \infty)$ and $\Delta \in(0,1)$.

Remark 4.1. Note that the condition

$$
\int_{D} \Phi(Q(x)) d m(x) \leq M
$$

implies (4.1). Thus, the condition (4.1) is more general than (4.3) and ring $Q$ homeomorphisms satisfying (4.3) form a subclass of $\Re_{M, \Delta}^{\Phi}$. Conversely, if the domain 
$D$ is bounded, then (4.1) implies the condition

$$
\int_{D} \Phi(Q(x)) d m(x) \leq M_{*}
$$

where $M_{*}=M \cdot\left(1+\delta_{*}^{2}\right), \delta_{*}=\sup _{x \in D}|x|$.

Proof. With no loss of generality we may assume that $\Phi(0)>0$. By Proposition 2.1 it is sufficient to show that mappings in $\mathfrak{R}_{M, \Delta}^{\Phi}$ are equicontinuous at every point $x_{0} \in D$. If $x_{0} \neq \infty$, then by the Proposition 2.2

$$
h\left(f(x), f\left(x_{0}\right)\right) \leq \frac{\alpha_{n}}{\Delta} \exp \left\{-\int_{\left|x-x_{0}\right|}^{\rho} \frac{d r}{r q_{x_{0}}^{\frac{1}{n-1}}(r)}\right\}
$$

for every fixed $x \in B\left(x_{0}, \rho\right)$ and every positive $\rho=\rho\left(x_{0}\right)<\operatorname{dist}\left(x_{0}, \partial D\right)$ where $q_{x_{0}}(r)$ is the mean value of $Q(x)$ over the sphere $\left|z-x_{0}\right|=r$ and $\alpha_{n}$ depends only on $n$. After the replacement $t=r / \rho$, we have that the integral from the right hand side in (4.5) is estimated by Lemma 3.1 in the following way

$$
\int_{\left|x-x_{0}\right|}^{\rho} \frac{d r}{r q_{x_{0}}^{\frac{1}{n-1}}(r)}=\int_{\varepsilon}^{1} \frac{d t}{t q^{\frac{1}{n-1}}(t)} \geq \frac{1}{n} \int_{e M(\varepsilon)}^{\frac{M(\varepsilon)}{\varepsilon^{n}}} \frac{d \tau}{\tau\left[\Phi^{-1}(\tau)\right]^{\frac{1}{n-1}}}
$$

where $\varepsilon=\left|x-x_{0}\right| / \rho, q(t)=q_{x_{0}}(\rho t)$ and

$$
M(\varepsilon)=f_{R} \Phi(Q(z)) d m(z)=\frac{1}{\Omega_{n} \rho^{n}\left(1-\varepsilon^{n}\right)} \int_{R} \Phi(Q(z)) d m(z)
$$

where $R=\left\{z \in \mathbf{R}^{n}:\left|x-x_{0}\right|<\left|z-x_{0}\right|<\rho\right\}$ is a ring centered at $x_{0}$ and $\Omega_{n}$ is the volume of the unit ball $\mathbf{B}^{n}$ in $\mathbf{R}^{n}$. Note that

$$
M(\varepsilon) \leq \frac{\beta_{n}\left(x_{0}\right)}{\Omega_{n}\left(1-\varepsilon^{n}\right)} \int_{R} \Phi(Q(z)) \frac{d m(z)}{\left(1+|z|^{2}\right)^{n}}
$$

where $\beta_{n}\left(x_{0}\right)=\left(1+\left(\rho\left(x_{0}\right)+\left|x_{0}\right|\right)^{2}\right)^{n} / \rho^{n}\left(x_{0}\right)$ because $|z| \leq\left|z-x_{0}\right|+\left|x_{0}\right| \leq \rho\left(x_{0}\right)+$ $\left|x_{0}\right|$. Thus,

$$
\Phi(0) \leq M(\varepsilon) \leq \frac{2 \beta_{n}\left(x_{0}\right)}{\Omega_{n}} M
$$

if $\varepsilon \leq 1 / \sqrt[n]{2}$ and, in particular, if $\varepsilon \leq 1 / 2$. Consequently,

$$
h\left(f(x), f\left(x_{0}\right)\right) \leq \frac{\alpha_{n}}{\Delta} \exp \left\{-\frac{1}{n} \int_{\lambda_{n} \beta_{n}\left(x_{0}\right) M}^{\frac{\Phi(0) \rho^{n}\left(x_{0}\right)}{\left|x-x_{0}\right|^{n}}} \frac{d \tau}{\tau\left[\Phi^{-1}(r)\right]^{\frac{1}{n-1}}}\right\}
$$

for all $x$ such that $\left|x-x_{0}\right|<\rho\left(x_{0}\right) / 2$ where $\lambda_{n}=2 e / \Omega_{n}$ depends only on $n$. Thus, $f \in \mathfrak{R}_{M, \Delta}^{\Phi}$ are equicontinuous at the point $x_{0}$. The case $x_{0}=\infty$ is reduced to $x_{0}=0$ by the inversion with respect to the unit sphere $|x|=1$.

Corollary 4.1. Each of the conditions (2.5)-(2.10) for $p \in(0, n-1]$ implies equicontinuity and normality of the classes $\mathfrak{R}_{M, \Delta}^{\Phi}$ for all $M \in(0, \infty)$ and $\Delta \in(0,1)$. 
Given a function $\Phi:[0, \infty] \rightarrow[0, \infty], M>0$ and $\Delta>0, S_{M, \Delta}^{\Phi}$ denotes the class of all homeomorphisms $f$ of $D$ in the Sobolev class $W_{\text {loc }}^{1, n}$ with a locally integrable $K_{I}(x, f)$ such that $h\left(\overline{\mathbf{R}^{n}} \backslash f(D)\right) \geq \Delta$ and (4.1) holds for $Q(x)=K_{I}(x, f)$. Note that if $\Phi$ is non-decreasing, convex and non-constant on $[0, \infty)$, then (4.1) itself implies that $K_{I}(x, f) \in L_{\text {loc }}^{1}$. Note also that $S_{M, \Delta}^{\Phi} \subset \mathfrak{R}_{M, \Delta}^{\Phi}$, see e.g. Theorem 4.1 in [17]. Thus, we have the following consequence.

Corollary 4.2. Each of the conditions (2.5)-(2.10) for $p \in(0, n-1]$ implies equicontinuity and normality of the class $S_{M, \Delta}^{\Phi}$ for all $M \in(0, \infty)$ and $\Delta \in(0,1)$.

Remark 4.2. The given conditions (2.5)-(2.10) for $p=n-1$ are weakest that lead to equicontinuity (normality) of the classes $S_{M, \Delta}^{\Phi}$ and $\mathfrak{R}_{M, \Delta}^{\Phi}$, see Theorem 5.1 further. The most interesting of them is (2.7) that can be rewritten in the following form:

$$
\int_{\delta}^{\infty} \log \Phi(t) \frac{d t}{t^{n^{\prime}}}=\infty
$$

where $\frac{1}{n^{\prime}}+\frac{1}{n}=1$, i.e. $n^{\prime}=2$ for $n=2, n^{\prime}$ is strictly increasing in $n$ and $n^{\prime}=$ $n /(n-1) \rightarrow 1$ as $n \rightarrow \infty$. Note also that the condition (4.2), as well as (5.1) further, can be rewritten in the form

$$
\int_{\delta}^{\infty} \frac{d \tau}{\tau \Phi_{n-1}^{-1}(\tau)}=\infty, \quad \Phi_{n-1}(t):=\Phi\left(t^{n-1}\right) .
$$

\section{Necessary conditions for equicontinuity}

Theorem 5.1. If the classes $S_{M, \Delta}^{\Phi} \subset \mathfrak{R}_{M, \Delta}^{\Phi}$ are equicontinuous (normal) for a non-decreasing convex function $\Phi:[0, \infty] \rightarrow[0, \infty]$, all $M \in(0, \infty)$ and $\Delta \in(0,1)$, then

$$
\int_{\delta_{*}}^{\infty} \frac{d \tau}{\tau\left[\Phi^{-1}(\tau)\right]^{\frac{1}{n-1}}}=\infty
$$

for all $\delta_{*} \in\left(\tau_{0}, \infty\right)$ where $\tau_{0}:=\Phi(0)$.

It is evident that the function $\Phi(t)$ in Theorem 5.1 cannot be constant because in the contrary case we would have no real restrictions for $K_{I}$ except $\Phi(t) \equiv \infty$ when the classes $S_{M, \Delta}^{\Phi}$ are empty. Moreover, by the known criterion of convexity, see e.g. Proposition 5 in I.4.3 of [5], the slope $[\Phi(t)-\Phi(0)] / t$ is nondecreasing. Hence the proof of Theorem 5.1 follows from the next statement.

Lemma 5.1. Let a function $\Phi:[0, \infty] \rightarrow[0, \infty]$ be non-decreasing and

$$
\Phi(t) \geq C \cdot t^{\frac{1}{n-1}} \quad \forall t \in[T, \infty]
$$

for some $C>0$ and $T \in(0, \infty)$. If the classes $S_{M, \Delta}^{\Phi} \subset \mathfrak{R}_{M, \Delta}^{\Phi}$ are equicontinuous (normal) for all $M \in(0, \infty)$ and $\Delta \in(0,1)$, then (5.1) holds for all $\delta_{*} \in\left(\tau_{0}, \infty\right)$ where $\tau_{0}:=\Phi(+0)$. 
Remark 5.1. As well-known, the critical exponent $n-1$ takes a key part in many problems of space mappings. The condition (5.2) can be rewritten in the form

$$
\Phi_{n-1}(t) \geq C \cdot t \quad \forall t \in[T, \infty]
$$

where $\Phi_{n-1}(t)=\Phi\left(t^{n-1}\right)$ and $C>0, T \in(0, \infty)$ that once more accentuates the significance of the function $\Phi_{n-1}$ in the question. In fact, it suffices also to require the weaker condition of convexity of $\Phi_{n-1}$ instead of $\Phi$ in Theorem 5.1.

Proof of Lemma 5.1. Let us assume that (5.1) is not true, i.e.

$$
\int_{\delta_{0}}^{\infty} \frac{d \tau}{\tau \Phi_{n-1}^{-1}(\tau)}<\infty
$$

for some $\delta_{0} \in\left(\tau_{0}, \infty\right)$ where $\Phi_{n-1}(t):=\Phi\left(t^{n-1}\right)$. Then also

$$
\int_{\delta}^{\infty} \frac{d \tau}{\tau \Phi_{n-1}^{-1}(\tau)}<\infty \quad \forall \delta \in\left(\tau_{0}, \infty\right)
$$

because $\Phi^{-1}(\tau)>0$ for all $\tau>\tau_{0}$ and $\Phi^{-1}(\tau)$ is non-decreasing. Note that by (5.2)

$$
\Phi_{n-1}(t) \geq C \cdot t \quad \forall t \geq T
$$

under some $C>0$ and $T \in(1, \infty)$. Furthermore, applying the linear transformation $\alpha \Phi+\beta$ with $\alpha=1 / C$ and $\beta=T$, see e.g. (2.7), we may assume that

$$
\Phi_{n-1}(t) \geq t \quad \forall t \in[0, \infty) .
$$

Of course, we may also assume that $\Phi(t)=t$ for all $t \in[0,1)$ because the values of $\Phi$ in $[0,1)$ give no information on $K_{I}(x, f) \geq 1$ in (4.1). It is clear that (5.5) implies $\Phi(t)<\infty$ for all $t<\infty$, see the criterion (2.7), cf. (2.10).

Now, note that the function $\Psi(t):=t \Phi_{n-1}(t)$ is strictly increasing, $\Psi(1)=\Phi(1)$ and $\Psi(t) \rightarrow \infty$ as $t \rightarrow \infty$. Hence the functional equation

$$
\Psi(K(r))=\left(\frac{\gamma}{r}\right)^{2} \quad \forall r \in(0,1]
$$

where $\gamma=\Phi^{1 / 2}(1) \geq 1$, is well solvable with $K(1)=1$ and a strictly decreasing continuous $K(r), K(r)<\infty, r \in(0,1]$, and $K(r) \rightarrow \infty$ as $r \rightarrow 0$. Taking the logarithm in (5.8), we have that

$$
\log K(r)+\log \Phi_{n-1}(K(r))=2 \log \frac{\gamma}{r}
$$

and by (5.7) we obtain that

i.e.,

$$
\log K(r) \leq \log \frac{\gamma}{r}
$$

Then by (5.8)

and by $(2.3)$

$$
K(r) \leq \frac{\gamma}{r}
$$

$$
\Phi_{n-1}(K(r)) \geq \frac{\gamma}{r}
$$

$$
K(r) \geq \Phi_{n-1}^{-1}\left(\frac{\gamma}{r}\right)
$$


It is sufficient to consider the case $D=\mathbf{B}^{n}$. We define the following mappings in the punctured unit ball $\mathbf{B}^{n} \backslash\{0\}$ :

$$
f(x)=\frac{x}{|x|} R(|x|), \quad f_{m}(x)=\frac{x}{|x|} R_{m}(|x|), \quad m=1,2, \ldots
$$

where

$$
\begin{gathered}
R(t)=\exp \{I(0)-I(t)\}, \quad R_{m}(t)=\exp \left\{I(0)-I_{m}(t)\right\}, \\
I(t)=\int_{t}^{1} \frac{d r}{r K(r)}, \quad I_{m}(t)=\int_{t}^{1} \frac{d r}{r K_{m}(r)}
\end{gathered}
$$

and

$$
K_{m}(r)= \begin{cases}K(r), & \text { if } r \geq 1 / m \\ K\left(\frac{1}{m}\right), & \text { if } r \in(0,1 / m)\end{cases}
$$

By (5.10)

$$
I(0)-I(t)=\int_{0}^{t} \frac{d r}{r K(r)} \leq \int_{0}^{t} \frac{d r}{r \Phi_{n-1}^{-1}\left(\frac{\gamma}{r}\right)}=\int_{\frac{\gamma}{t}}^{\infty} \frac{d \tau}{\tau \Phi_{n-1}^{-1}(\tau)} \quad \forall t \in(0,1]
$$

where $\gamma / t \geq \gamma \geq 1>\Phi(0)=0$. Hence by the condition (5.5)

$$
I(0)-I(t) \leq I(0)=\int_{0}^{1} \frac{d r}{r K(r)}<\infty \quad \forall t \in(0,1] .
$$

Moreover, $f_{m}$ and $f \in C^{1}\left(\mathbf{B}^{n} \backslash\{0\}\right)$ because $K_{m}(r)$ and $K(r)$ are continuous, and hence locally quasiconformal in $\mathbf{B}^{n} \backslash\{0\}$. Furthermore, $f_{m}$ are $K_{m}$-quasiconformal in $\mathbf{B}^{n}$ where $K_{m}=K(1 / m), f_{m}(0)=0$.

Next, the tangent and radial distortions under the mapping $f$ on the sphere $|x|=\rho, \rho \in(0,1)$, are easy calculated

$$
\begin{aligned}
& \delta_{\tau}(x)=\frac{|f(x)|}{|x|}=\frac{\exp \left\{\int_{0}^{\rho} \frac{d t}{t K(t)}\right\}}{\rho}, \\
& \delta_{r}(x)=\frac{\partial|f(x)|}{\partial|x|}=\frac{\exp \left\{\int_{0}^{\rho} \frac{d t}{t K(t)}\right\}}{\rho K(\rho)}
\end{aligned}
$$

and we see that $\delta_{\tau}(x) \geq \delta_{r}(x)$ because $K(r) \geq 1$. Consequently, by the spherical symmetry we have that

$$
K_{I}(x, f)=\frac{\delta_{\tau}^{n-1}(x) \cdot \delta_{r}(x)}{\delta_{r}^{n}(x)}=K^{n-1}(|x|)
$$

at all points $x \in \mathbf{B}^{n} \backslash\{0\}$, see e.g. Subsection I.4.1 in [20]. Note that

$$
f_{m}(x) \equiv f(x) \quad \forall x: \frac{1}{m}<|x|<1, m=1,2 \ldots
$$


Hence it is similarly calculated $K_{I}\left(x, f_{m}\right)=K_{I}(x, f)=K^{n-1}(|x|)$ for $\frac{1}{m}<|x|<1$ and $K_{I}\left(x, f_{m}\right)=K(1 / m)$ for $0<|x|<\frac{1}{m}$. Thus, $f_{m}$ are quasiconformal in $\mathbf{B}^{n}$, hence $f_{m} \in W_{l o c}^{1, n}$ and by $(5.8)$

$$
\begin{aligned}
& \int_{\mathbf{B}^{n}} \Phi\left(K_{I}\left(x, f_{m}\right)\right) d m(x) \leq \int_{\mathbf{B}^{n}} \Phi_{n-1}(K(|x|)) d m(x) \\
& =\omega_{n-1} \int_{0}^{1} \frac{\Psi(K(r))}{r K(r)} \cdot r^{n} d r \leq \gamma^{2} \omega_{n-1} \int_{0}^{1} \frac{d r}{r K(r)} \leq M:=\gamma^{2} \omega_{n-1} I(0)<\infty .
\end{aligned}
$$

Note that $f_{m}$ map the unit ball $\mathbf{B}^{n}$ onto the ball centered at the origin with the radius $e^{I(0)}<\infty$. Thus, $f_{m} \in S_{M, \Delta}^{\Phi}$ with $M$ given above and some $\Delta>0$.

On the other hand, it is easy to see that

$$
\lim _{x \rightarrow 0}|f(x)|=\lim _{t \rightarrow 0} \rho(t)=e^{0}=1,
$$

i.e. $f$ maps the punctured ball $\mathbf{B}^{n} \backslash\{0\}$ onto the ring $1<|y|<e^{I(0)}$. Then by (5.12) and (5.13) we obtain that

$$
\left|f_{m}(x)\right|=|f(x)| \geq 1 \quad \forall x:|x| \geq 1 / m, m=1,2, \ldots,
$$

i.e. the family $\left\{f_{m}\right\}_{m=1}^{\infty}$ is not equicontinuous at the origin. The contradiction disproves the assumption (5.4).

Remark 5.2. Theorem 5.1 shows that the condition (4.2) in Theorem 4.1 is not only sufficient but also necessary for equicontinuity (normality) of classes with the integral constraints of the type either (4.1) or (4.4) with a convex non-decreasing $\Phi$. In view of Proposition 2.3, the same concerns to all the conditions (2.5)-(2.10) with $p=n-1$.

Corollary 5.1. The equicontinuity (normality) of the classes $S_{M, \Delta}^{\Phi} \subset \mathfrak{R}_{M, \Delta}^{\Phi}$ for all $M \in(0, \infty), \Delta \in(0,1)$ and a non-decreasing convex $\Phi$ implies that

$$
\int_{\delta}^{\infty} \log \Phi(t) \frac{d t}{t^{n^{\prime}}}=\infty
$$

for all $\delta>t_{0}$, where $t_{0}:=\sup _{\Phi(t)=0} t, t_{0}=0$ if $\Phi(0)>0, \frac{1}{n^{\prime}}+\frac{1}{n}=1$, i.e. $n^{\prime}=n /(n-1)$.

Recall that by Remark 4.2 and Proposition 2.3 the condition (5.14) is also sufficient for equicontinuity (normality) of the classes $S_{M, \Delta}^{\Phi}$ and $\mathfrak{R}_{M, \Delta}^{\Phi}$.

\section{References}

[1] Ahlfors, L.: On quasiconformal mappings. - J. Anal. Math. 3, 1953/54, 1-58.

[2] Andreian Cazacu, C.: On the length-area dilatation. - Complex Var. Theory Appl. 50:7-11, 2005, 765-776.

[3] Biluta, P. A.: Extremal problems for mappings which are quasiconformal in the mean. Sibirsk. Mat. Zh. 6, 1965, 717-726.

[4] Bishop, C. J., V. Ya. Gutlyanskit, O. Martio, and M. Vuorinen: On conformal dilatation in space. - Int. J. Math. Math. Sci. 22, 2003, 1397-1420.

[5] Bourbaki, N.: Functions of a real variable. - Springer, Berlin, 2004. 
[6] Gehring, F. W.: Rings and quasiconformal mappings in space. - Trans. Amer. Math. Soc. 103, 1962, 353-393.

[7] Golberg, A.: Homeomorphisms with finite mean dilatations. - Contemp. Math. 382, 2005, $177-186$.

[8] Gutlyanskit, V., O. Martio, T. Sugawa, and M. Vuorinen: On the degenerate Beltrami equation. - Trans. Amer. Math. Soc. 357, 2005, 875-900.

[9] Iwaniec, T., and G. Martin: Geometric function theory and nonlinear analysis. - Clarendon Press, Oxford, 2001.

[10] Iwaniec, T., and G. Martin: The Beltrami equation. - Mem. Amer. Math. Soc. 191, 2008, $1-92$.

[11] Kruglikov, V. I.: Capacities of condensors and quasiconformal in the mean mappings in space. - Mat. Sb. 130:2, 1986, 185-206.

[12] Krushkal', S. L.: On mappings that are quasiconformal in the mean. - Dokl. Akad. Nauk SSSR 157:3, 1964, 517-519.

[13] KrushKaL', S. L., and R. KÜHNAU: Quasiconformal mappings, new methods and applications. - Novosibirsk, Nauka, 1984 (in Russian).

[14] KUD'YAVIN, V.S.: Behavior of a class of mappings quasiconformal in the mean at an isolated singular point. - Dokl. Akad. Nauk SSSR 277:5, 1984, 1056-1058.

[15] KÜHnau, R.: Über Extremalprobleme bei im Mittel quasiconformen Abbildungen. - Lecture Notes in Math. 1013, 1983, 113-124 (in German).

[16] Lehto, O., and K. Virtanen: Quasiconformal mappings in the plane. - Springer, New York, 1973.

[17] Martio, O., V. Ryazanov, U. Srebro, and E. Yakubov: Moduli in modern mapping theory. - Springer, New York, 2009.

[18] Perovich, M.: Isolated singularity of the mean quasiconformal mappings. - Lecture Notes in Math. 743, 1979, 212-214.

[19] Pesin, I. N.: Mappings quasiconformal in the mean. - Dokl. Akad. Nauk SSSR 187:4, 1969, 740-742.

[20] Reshetnyak, Yu. G.: Space mappings with bounded distortion. - Transl. Math. Monogr. 73, Amer. Math. Soc., 1989.

[21] Ryazanov, V. I.: On mappings that are quasiconformal in the mean. - Sibirsk. Mat. Zh. 37:2, 1996, 378-388.

[22] Ryazanov, V., and E. A. Sevost'yanov: Toward the theory of ring $Q$-homeomorphisms. Israel J. Math. 168, 2008, 101-118.

[23] Ryazanov, V., U. Srebro, and E. Yakubov: On ring solutions of Beltrami equation. - J. Anal. Math. 96, 2005, 117-150.

[24] Ryazanov, V., U. Srebro, and E. Yakubov: On integral conditions in the mapping theory. - Ukr. Math. Bull. 7, 2010, 73-87.

[25] Ukhlov, A., and Vodopyanov, S. K.: Mappings associated with weighted Sobolev spaces. In: Complex analysis and dynamical systems III, Contemp. Math. 455, 2008, 369-382.

[26] VÄISÄLÄ, J.: Lectures on $n$-dimensional quasiconformal mappings. - Lecture Notes in Math. 229, Springer-Verlag, Berlin, 1971. 